\title{
HOLOMORPHIC APPROXIMATION TO BOUNDARY VALUE ALGEBRAS
}

\author{
BY FRANK T. BIRTEL ${ }^{1}$
}

0. Introduction. The topic of this lecture is the well-known question: Letting $\mathrm{Hol}(X)$ denote the algebra of functions holomorphic in a neighborhood of $X$, for which relatively compact domains $D$ in $\mathbf{C}^{n}$ does the injection

$$
\operatorname{Hol}(\bar{D}) \hookrightarrow \operatorname{Hol}(D) \cap C(\bar{D})
$$

have uniformly dense range? In terms of uniform algebras, it becomes a question of necessary and sufficient conditions for the uniform algebra $H(\bar{D})$ of uniform limits of functions holomorphic near $\bar{D}$ to coincide with the uniform algebra $A(\bar{D})$ of all continuous functions on $\bar{D}$ which are holomorphic on $D$.

A necessary condition obviously is that the respective collections of complex homomorphisms, $\Delta H(\bar{D})$ and $\Delta A(\bar{D})$, of these algebras coincide. Since Arens [1] demonstrated for the case $n=1$ that $\Delta A(\bar{D})=\bar{D}$ (i.e. every complex homomorphism of $A(\bar{D})$ arises from point evaluation at points of $\bar{D})$, and again in the case $n=1$ Runge's theorem implies $\Delta H(\bar{D})=\bar{D}$, the necessary condition always holds on domains in the complex plane. Nevertheless, there exist relatively compact connected domains $D$ in the complex plane for which $H(\bar{D}) \neq A(\bar{D})$. Several such examples are developed in Gamelin's treatise [17]. Failure of approximation in the plane is a local boundary property of $\bar{D}$, since $H(\bar{D})=A(\bar{D})$, if for every $z \in \bar{D}$ there is a closed neighborhood $V_{z}$ on which $A\left(\vec{D} \cap V_{z}\right)=H\left(\bar{D} \cap V_{z}\right)$. See [17]. In 1967, Vitushkin [32] formulated a necessary and sufficient condition for approximation in terms of continuous analytic capacity; roughly speaking, approximation takes place if the boundary of $D$ is "not too large."

Following this one-dimensional motivation, it has frequently been conjectured that $\operatorname{Hol}(\bar{D}) \hookrightarrow \operatorname{Hol}(D) \cap C(\bar{D})$ has dense range on any relatively compact domain of holomorphy $D$ with "reasonable" boundary. Recent examples, which are described below, show the conjecture to be false even in the case $\partial D$ is $C^{(\infty)}$. These counterexamples are established by showing the failure of the necessary condition-viz., $\Delta \underline{H}(\bar{D}) \neq \Delta A(\bar{D})$, due to analytic continuation beyond $\bar{D}$ of functions in $H(\bar{D})$ not possible to the same extent for functions in $A(\bar{D})$. When $\bar{D}$ is holomorphically convex-i.e. $\Delta H(\bar{D})=\bar{D}$,

An address delivered at the Columbia, South Carolina meeting of the Society on November 19, 1976; received by the editors September 12, 1977.

AMS (MOS) subject classifications (1970). Primary 32E25, 32E30; Secondary 32D10, 32D15, 32F15, 46J10.

${ }^{1}$ The author was partially supported by NSF grant MCS77-01675. 
simultaneous analytic continuation is not possible beyond $\bar{D}$ for all functions in $H(\bar{D})$. Without the condition of boundary smoothness, examples in which $\Delta H(\bar{D})=\Delta A(\bar{D})$, but $H(\bar{D}) \neq A(\bar{D})$ have been known for some time. The latter type of counterexample does not simply perpetuate the one-dimensional type abnormalities, for the boundaries in these examples may fail to be smooth on a very small set-even a point; difficulties arise through failure of the segment property at certain boundary points.

Definition 1. D has the segment property at $z \in \partial D$, if there exists a neighborhood $W$ of $z$ in $\bar{D}$ and $w \in C^{n}$ such that for all $0<t<1, W+t w \subseteq$ D.

Based on these vague comments, there is a form in which the original conjecture may survive.

BASIC CONJECTURE. If $D$ is a domain in $\mathbf{C}^{n}$ whose closure is holomorphically convex and which satisfies the segment property at each boundary point, then $H(\bar{D})=A(\bar{D})$.

The underlying purpose of this exposition is to fortify the reasonableness of the Basic Conjecture. The selection of topics which occurs in the process is not intended to slight equally valuable, but unmentioned, contributions to the subject of holomorphic approximation.

OUTLINE. For insight into the difficulties which obstruct approximation, a few provocative counterexamples are discussed in \$1. In \$2, the significance of the segment property in determining $\triangle A(\bar{D})$ and in providing an alternative description of $A(\vec{D})$ on a holomorphically convex set $\bar{D}$ is considered. $\$ 3$ describes a general method for handling approximation on strongly pseudoconvex domains and thereby sets the stage for dealing with domains with less amenable boundaries. The present status of the Basic Conjecture with respect to weakly pseudoconvex domains is the emphasis of $\$ 4$. Finally, in $\$ 5$, an attempt is made to formulate the question of approximation more generally and through this formulation to generate problems which remain unanswered even in the specific cases previously considered.

1. Counterexamples. Let $\Omega_{1}=\{(z, w):|z|<|w|<1\} \subseteq C^{2}$. Even though $\Omega_{1}$ is a domain of holomorphy, the interior of the intersection of the domains of holomorphy containing $\bar{\Omega}_{1}$ (i.e., the Nebenhülle $n\left(\Omega_{1}\right)$ of $\Omega_{1}$ ) is the bi-disc $D^{2}=\{(z, w):|z|<1,|w|<1\}$. Indeed, $\bar{\Omega}_{1}$ is not holomorphically convex. $\bar{\Omega}_{1}$ is the most elementary example of a domain of holomorphy on the closure of which approximation fails. The function $f$ defined by $f(z, w)=z^{2} / w$ extends continuously to the boundary singularity $(0, \underline{0})$ but does not extend holomorphically to $D^{2}$ as do all functions in $H\left(\bar{\Omega}_{1}\right)$. This example obscures the respective roles of holomorphic convexity and boundary smoothness. Actually, it is not disparity between the homomorphisms of the respective algebras, but rather holomorphic extension of functions in $H\left(\bar{\Omega}_{1}\right)$ to a neighborhood of the boundary singularity $(0,0)$, which accounts for the failure of approximation. However, this does underline the fact that in higher dimensions smoothness can fail on a "not too large" boundary without approximation occurring.

Since the main thrust of these remarks addresses domains with reasonable boundary, suffice it to note that examples provided by E. Kallin [23], 
Senichkin [27] and Sibony [30], show the existence of open relatively compact subsets $D$ of $\underline{\mathbf{C}}^{n}$ with the properties (i) $\Delta H(\bar{D})=\bar{D}=\Delta A(\bar{D})$; (ii) $\partial_{0} H(\bar{D})$ $=\partial D=\partial_{0} A(\bar{D})$, where " $\partial_{0}$ " denotes "the Šilov boundary of"; (iii) $A(\bar{D}) \neq$ $H(\bar{D})$; but (iv) $D$ fails to have the segment property somewhere at the boundary. These domains may even be connected and polynomially convex in contrast with the celebrated theorem of Mergelyan which asserts that every function in $A(\bar{D})$ is a uniform limit of polynomials, if $\bar{D} \subseteq \mathbf{C}$ and if $\bar{D}$ is polynomially convex. Also, one of Senichkin's examples has the property that the peak points of both algebras are the same although $\partial_{0} H(D) \neq \partial D$.

On the other hand, under the assumption that $D$ is a domain of holomorphy with smooth boundary, counterexamples to approximation still appeared unlikely until 1975 when Diederich and Fornaess [12] constructed a relatively compact domain of holomorphy $\Omega_{2}$ in $\mathbf{C}^{n}$ with $C^{\infty}$-boundary for which $H\left(\bar{\Omega}_{2}\right) \neq A\left(\bar{\Omega}_{2}\right)$. Approximation on $\Omega_{2}$ fails for the most elementary reason: failure to satisfy the necessary condition that $\Delta H\left(\bar{\Omega}_{2}\right)=\Delta A\left(\bar{\Omega}_{2}\right)$.

The domain $\Omega_{2}$ constructed by Diederich and Fornaess is not holomorphically convex, has a nontrivial Nebenhülle and, a fortiori, is not strongly pseudoconvex; i.e. there does not exist a smooth function $\rho$ in a neighborhood $U$ of $\partial \Omega_{2}$ with the properties:

(a) $\Omega_{2} \cap U=\{z \in U: \rho(z)<0\}$;

(b) $d \rho(z) \neq 0$ for all $z \in U$;

(c) the Levi form of $\rho$ at any point $z \in \partial \Omega_{2}$ is positive definite on $U$.

(In fact, they show that no such exhaustion function $\rho$ exists which is plurisubharmonic in $U$.) However, there is [9] a strictly plurisubharmonic exhaustion function which is Hölder continuous on the closure of any weakly pseudoconvex domain.

A brief description of the Diederich and Fornaess example follows. Let $\lambda$ : $R \rightarrow R^{+} \cup\{0\}$ be a "sufficiently" convex, smooth function, vanishing on the nonpositive reals and strictly positive on the positive reals. For large $R>1$, define $\rho_{R}$ by

$$
\rho_{R}(z, w)=|w+\exp (i \ln z \bar{z})|^{2}-1+\lambda\left(|z|^{-2}-1\right)+\lambda\left(|z|^{2}-R^{2}\right) .
$$

Then let

$$
\Omega_{2}=\left\{(z, w) \in(\mathbf{C}-\{0\}) \times \mathbf{C}: \rho_{R}(z, w)<0\right\} .
$$

The Levi-form of $\rho_{R}$ vanishes precisely on the annulus $A_{R}=\{(z, w): 1 \leqslant|z|$ $<R$ and $w=0\}$. Furthermore, $\Omega_{2}$ is weakly pseudoconvex, which is to assert that the Levi-form of $\rho_{R}$ is nonnegative on complex tangent vectors to $\partial \Omega_{2}$ : i.e.

$$
\sum_{i, j} \frac{\partial \rho_{R}}{\partial z_{i} \partial \bar{z}_{j}}(\zeta) w_{i} \bar{w}_{j} \geqslant 0, \quad \text { if } \sum_{i} \frac{\partial \rho_{R}}{\partial z_{i}}(\zeta) w_{i}=0 \text { for all } \zeta \in \partial \Omega_{R} .
$$

Strongly pseudoconvex boundary points-boundary points where the Levi form is positive-are peak points for the continuous boundary value algebra $A\left(\bar{\Omega}_{2}\right)$. Furthermore, Hakim and Sibony [18] show that for any weakly pseudoconvex domain $D, \partial_{0} A(\bar{D})$ is the closure of the set of strongly pseudoconvex boundary points. By applying Hartog's theorem one can see that every holomorphic function in $\Omega_{2}$ continues holomorphically to 


$$
\tilde{\Omega}_{2}=\Omega_{2} \cup\left\{(z, w): e^{\pi}<|z|<e^{2 \pi} \text { and }|w|<2\right\} .
$$

Since $\tilde{\Omega}_{2}$ contains peak points of $A\left(\bar{\Omega}_{2}\right)$ in a neighborhood of which functions in $H\left(\bar{\Omega}_{2}\right)$ can be continued, the two uniform algebras must be distinct.

Thus the possibility of holomorphic approximation on a domain $D$ with nice boundary seems to be related to holomorphic convexity or to the existence of a plurisubharmonic defining function for $D$ with special properties beyond $\bar{D}$.

2. Homomorphism spaces. Holomorphically convex compact sets $K$ in $\mathbf{C}^{n}$ can be characterized as the intersection of the natural projections into $\mathbf{C}^{n}$ of the envelopes of holomorphy of a fundamental system of open neighborhoods of $K$. (See Birtel [6] or, for a more detailed presentation of the proof, Birtel [4].) Furthermore, the interior of a holomorphically convex set, and, a fortiori, of a holomorphic set, is a domain of holomorphy [4]. In the presence of holomorphic convexity it is natural to seek conditions to guarantee that the homomorphism space of the continuous boundary value algebra is no larger. This was verified by Hakim and Sibony [18] for a domain $D \subseteq \mathbf{C}^{n}$ such that (i) $D$ is weakly pseudoconvex, (ii) $D$ has the segment property at each $z \in \partial D$, and (iii) $\bar{D}$ is a compact holomorphic set (i.e. an intersection of Stein neighborhoods). If (i), (ii) and (iii) hold for $D$, then $\Delta H(\bar{D})=\Delta A(\bar{D})=\bar{D}$ and $\partial_{0} H(\bar{D})=\partial_{0} A(\bar{D})$, which is the closure of the strongly pseudoconvex points in the case $\partial D$ is $C^{(2)}$. More recently, Beatrous [2] has extended these results to holomorphically convex sets $X$ which may fail to have the segment property on a "sufficiently small" subset of the boundary. Specifically he shows:

Theorem 2.1. Let $A$ and $B$ be uniform algebras on $X=\Delta A$ with $A \subseteq B$. Suppose $N \subseteq X$ is closed and $B$ consists of functions which are uniform limits of continuous functions on $X$, locally approximable on $X \backslash N$ by restrictions of functions in $A$. Under these conditions, if $\Delta B \neq X$, then $\partial_{0} B_{(\Delta B \backslash X)^{-}} \subseteq N$, where $B_{(\Delta B \backslash X)^{-}}$is the algebra of uniform limits on $(\Delta B \backslash X)^{-}$of restrictions of functions in $B$. In particular, if $\Delta B_{N} \subseteq X$, then $\Delta B=X$.

An immediately applicable situation arises when $\bar{D}$ is holomorphically convex, $N$ is the set of boundary points of $D$ at which the segment property fails and $A=H(\bar{D})$. Since at each $\rho \in \partial D$ at which the segment property holds there exists a neighborhood $U$ of $\rho$ in $\bar{D}$ such that the restrictions map $\operatorname{Hol}(\bar{D}) \rightarrow A(\bar{D})_{\bar{U}}$ has dense range, more generally $N$ may be regarded as any closed subset of $\partial D$ which contains the set of points in $\partial D$ at which local approximation by functions in $\operatorname{Hol}(\bar{D})$ fails to occur. In the examples of Sibony [30] and Kallin [23], cited earlier, the conditions of Theorem 2.1 are fulfilled; for in each of these examples, $\Delta A(\bar{D})_{N} \subseteq \bar{D}$.

Proof of Theorem 2.1. (Beatrous). Define $\pi: \Delta B \rightarrow X$ by $\pi(\phi)=\phi \mid A$ and for $f \in C(X)$, let $\tilde{f}=f \circ \pi$. If the theorem is false, there exists $x_{0} \in X \backslash$ $N$ which is a strong boundary point for the uniform algebra $B_{\Delta B \backslash X}$. There is a neighborhood $U$ of $x_{0}$ such that, if $f$ is continuous on $\Delta B \backslash \pi^{-1}(N)$ and locally approximable there by functions in $\hat{B}$ and if $f \mid X \equiv 0$, then $f \mid U \equiv 0$. Now any $h \in B$ can be realized as the limit of functions $h_{n}$ continuous on $X$ and locally approximable on $X \backslash N$ by functions in $A$. Thus $\tilde{h}_{n} \rightarrow \tilde{h}$ uniformly 
on $\Delta B$. With $\hat{h}_{n}$ denoting the extension of $h_{n}$ to $\Delta B, \tilde{h}_{n}=\hat{h}_{n}$ on $U$, since $\tilde{h}_{n}=\hat{h}_{n}$ on $X$. Therefore, $\tilde{h}=\hat{h}$ on $U$ for all $h \in B$. This implies $\pi \mid U$ is one-to-one so $U \subseteq X$. But $U$ is a neighborhood of $x_{0} \in(\Delta B \backslash X)^{-}$, which is a contradiction.

Encouragingly, the Basic Conjecture is sustained by these considerations. At least holomorphic approximation cannot fail due to the presence of new complex homomorphisms, whenever the closure of the domain is holomorphically convex and satisfies the segment property at each boundary point.

3. Strongly pseudoconvex domains. All is well for these domains. Strongly pseudoconvex domains with smooth boundary have closures which are holomorphic sets, hence are holomorphically convex, and satisfy the segment property at each boundary point. For $D$ strongly pseudoconvex with various assumptions about boundary smoothness, the result that $H(\bar{D})=A(\bar{D})$ appears in Kerzmann [24], Henkin [20], Lieb [25] and Fornaess [15].

For products of strongly pseudoconvex domains with smooth boundaries, the theorem has been established by Weinstock [33] and Sibony [28]. All of these results can be subsumed under a general procedure which may ultimately have a broader application. This general approach is described below.

The fundamental observation first suggested to the author by Reese Harvey is

The BASIC Lemma. IF, whenever $\mu$ is a measure supported by $\bar{D}$ which annihilates functions in $H(\bar{D})$, there exists a current $\omega$ of bi-degree $(n, n-1)$ with measure coefficients supported in $\bar{D}$ such that $\bar{\partial} \omega=\mu$ in the sense of distributions; THEN $H(\bar{D})=A(\bar{D})$, provided that $D$ has the segment property at each boundary point.

Note. In the complex plane the current $\omega$ of the above lemma is given by $(1 / \pi) \hat{\mu}(z) d z$, where $\hat{\mu}$ is the Cauchy transform of the orthogonal measure $\mu$.

OUTLINE OF THE PROOF. Let $\phi$ be a partition of unity subordinate to a cover of $\bar{D}$. Then $\bar{\partial}(\phi \omega)=\bar{\partial} \phi \wedge \omega+\phi \bar{\partial} \omega$ is a measure. If $\operatorname{spt}(\phi) \subseteq D$, then $\bar{\partial}(\phi \omega)(g)=\phi \omega(\bar{\partial} g)=0$ for all $g \in A(\bar{D})$. If $\operatorname{spt}(\phi) \cap \partial D \neq \varnothing$, then it may be assumed that the cover was so chosen that $\operatorname{spt}(\phi) \subseteq U$ and for some $\omega \in \mathbf{C}^{n}$ and for $0<t<1, z+t \omega \in D$ for all $z \in \bar{D} \cap U$. Let $(\phi \omega)_{t}$ denote the push forward of $\phi \omega$ by this affine map. Then $(\phi \omega)_{t} \rightarrow \phi \omega$ and $\bar{\partial}(\phi \omega)_{t}(g)=$ $(\phi \omega)_{t}(\bar{\partial} g)=0$ for all $g \in A(\bar{D})$. Therefore $\overline{\partial \omega}=\mu$ annihilates $A(\bar{D})$ or, equivalently, $H(\bar{D})=A(\bar{D})$.

The simplest circumstance in which the hypotheses of the Basic Lemma are verifiable is when $D$ is strongly pseudoconvex. Then $\bar{D}=\bigcap_{k=1}^{\infty} \Omega_{k}$ with $\Omega_{k+1} \subset \subset \Omega_{k}$ and $\Omega_{k}$ a domain of holomorphy for $k=1,2, \ldots$ By wellknown results of Dieudonné and Schwartz for each $\mu \in \operatorname{Hol}\left(\Omega_{k}\right)$ there exists an $(n, n-1)$ current $\lambda_{k}$ with $\bar{\partial} \lambda_{k}=\mu$ and $\lambda_{k}$ compactly supported in $\Omega_{k}$. In addition, the inhomogeneous Cauchy-Riemann equation can be solved boundedly on $\Omega_{k}$. So for each closed $C^{(\infty)}$ differential form $\alpha$ of bi-degree $(0,1)$ on $\Omega_{k}$,

$$
\left|\lambda_{k}(\alpha)\right|=\left|\lambda_{k}(\bar{\partial} f)\right|=\left|\bar{\partial} \lambda_{k}(f)\right| \leqslant\|\mu\|\|f\|_{\Omega_{k}} \leqslant\|\mu\| C_{k}\|\alpha\|_{\Omega_{k}} .
$$

By Hahn-Banach extend $\lambda_{k}$ with no greater norm to all $C^{(\infty)}$ forms of 
bi-degree $(0,1)$ to get a current $\omega_{k}$ with measure coefficients satisfying: (i) $\bar{\partial} \omega_{k}=\mu$, (ii) $\operatorname{spt}\left(\omega_{k}\right) \subseteq \Omega_{k}$ and $\left\|\omega_{k}\right\| \leqslant C_{k}$. Since the fundamental system $\left\{\Omega_{k}\right\}$ of neighborhoods can be chosen as small perturbations of $D$, it is possible to obtain a constant $C$ such that $C_{k} \leqslant C$ for all $k$. The proof is designed to apply whenever this condition holds. Then by the BourbakiAlaoglu theorem there exists a current $\omega$ in the weak-star closure of $\left\{\omega_{k}\right\}$ which fulfills the requirements of the Basic Lemma.

This argument can be simplified by using the explicit nature of the kernel which solves the inhomogeneous Cauchy-Riemann equation; the slightly more complicated formulation here avoids any specific choice of the kernel and hence could conceivably apply in more general situations.

A presumably weaker version of the Basic Lemma suffices to manufacture a current $\omega$ with the desired properties. If $\mu$ is orthogonal to $\operatorname{Hol}(\bar{D})$ and $\operatorname{spt}(\mu) \subseteq \bar{D}$, then the existence of a flat current $\Lambda$ of bi-degree $(n, n-1)$ with $\operatorname{spt}(\Lambda) \subseteq \bar{D}, \bar{\partial} \Lambda=\mu$ and flat norm $F_{\bar{D}}(\Lambda)<\infty$ implies the existence of a current $\omega$ of finite mass (measure coefficients) with the desired properties. For the definition of flat current, see Federer [14]. The characterization of flat currents $[14$, p. 375$]$ gives the existence of ${ }^{\mathcal{L}^{n}}$-summable currents $\lambda_{\delta}$ and $\nu_{\delta}$ supported in $\bar{D}_{\delta}=\{z: \operatorname{dist}(z, \bar{D}) \leqslant \delta\}$ with the properties that

(i) $\Lambda=\lambda_{\delta}+d v_{\delta}, \operatorname{spt}\left(\lambda_{\delta}\right) \subseteq \bar{D}_{\delta}, \operatorname{spt}\left(\nu_{\delta}\right) \subseteq \bar{D}_{\delta}$,

(ii) $\int\left(\left\|\lambda_{\delta}\right\|+\left\|\nu_{\delta}\right\|\right) d \mathcal{L}^{n} \leqslant F_{\bar{D}}(\Lambda)+\delta$.

But $\mu=\partial \Lambda=d \Lambda=d \lambda_{\delta}+d d v_{\delta}=d \lambda_{\delta}=\partial \lambda_{\delta}$. So $\left\{\lambda_{\delta}: \delta>0\right\}$ is a collection of uniformly bounded $\mathfrak{L}^{n}$-summable $(n, n-1)$ normal currents with $\operatorname{spt}\left(\lambda_{\delta}\right)$ $\subseteq \bar{D}_{\delta}$. Without loss of generality, assume $\lambda_{\delta}$ converges to $\lambda$ by using the compactness theorem for normal currents $[14, p .414]$. Then $\lambda$ is $\varrho^{n}$-summable $(n, n-1)$ current supported in $\bar{D}$ and $\bar{\partial} \lambda=\mu$. Whether this observation will ultimately be useful is still unanswered. The proposition does seem to bear relation to the work of Cole and Range [8].

The crucial result which makes application of the Basic Lemma possible is the availability of a uniformly bounded family of solutions to the inhomogeneous Cauchy-Riemann equations on a fundamental system of neighborhoods of the closure of the given domain. In all other proofs of approximation similar uniform bounds are critical to the success of the demonstrations. Of course, control of the constants $C_{k}$ depends upon detailed information about the kernel which is used to construct bounded solutions to the inhomogeneous Cauchy-Riemann equation.

Finally, Siu and Range [26] exhibit kernels which do the job and provide the ingredients for proving holomorphic approximation on domains with piecewise smooth boundaries which are relatively compact normal intersections of strongly pseudoconvex domains.

4. Weakly pseudoconvex domains. On a strongly pseudoconvex domain the existence of a smooth strictly plurisubharmonic defining function provides the means for constructing kernels to obtain uniformly bounded solutions of the inhomogeneous Cauchy-Riemann equations which are uniformly bounded on a fundamental system of strongly pseudoconvex neighborhoods gotten by small perturbations of the boundary of the original domain. Unlike strongly pseudoconvex domains, weakly pseudoconvex domains with smooth 
boundary do not, in general, have "nice" defining functions, are not always intersections of strongly pseudoconvex domains and, even when they are, do not seem to allow for control of the uniform bounds for solutions of the inhomogeneous Cauchy-Riemann equations near their closures. Therefore, the ingredients for application of the Basic Lemma in settling the question of holomorphic approximation are not available, even if the closure of the domain is known to be a holomorphic set.

In the weakly pseudoconvex case, the approach has been to apply what is known for strongly pseudoconvex domains near the strongly pseudoconvex boundary points and to assume certain geometric conditions near the weakly pseudoconvex points so that holomorphic approximation holds near each of the two types of boundary points, and then to patch approximates in order to obtain a global approximation.

LEMMA 4.1. Let $D$ be a relatively compact domain in $\mathbf{C}^{n}$ with $C^{(4)}$ boundary. Suppose $\bar{D}$ is holomorphically convex. Let $s(\bar{D})$ be the set of strongly pseudoconvex boundary points and let $N_{1}, N_{2}$ be open sets in $\mathbf{C}^{n}$ with $N_{1} \subset \subset N_{2}$ and $N_{2} \cap \partial D \subset \subset s(\bar{D})$. Then there is a neighborhood $\tilde{D}$ of $\bar{D}$ and a constant $k$ with the property:

If $D^{\prime} \supset D$ is a sufficiently small $C^{(4)}$ perturbation of $D$ and if $\alpha$ is a $\bar{\partial}$-closed $C^{(\infty)}$ differential form of bi-degree $(0,1)$ with $\operatorname{spt}(\alpha) \subseteq N_{1}$, then there is a $C^{(\infty)}$ function $u$ on $D^{\prime} \cup\left(\tilde{D} \backslash N_{2}\right)$ with $\overline{\partial u}=\alpha$ on $D^{\prime}, \bar{\partial} u=0$ on $\tilde{D} \backslash N_{2}$, and $\|u\|_{\bar{D}} \leqslant k\|\alpha\|_{\bar{D}^{\prime}}$

A version of this lemma appears in [35]; the above formulation for holomorphically convex sets is due to Beatrous [2].

Lemma 4.1 localizes those properties of strongly pseudoconvex domains which are most relevant for approximation; hence, not unexpectedly, Beatrous can show

THeORem 4.2. Let $D$ satisfy the conditions of Lemma 4.1 and let $E=\partial D \backslash$ $s(\bar{D})$. Then a function $f \in A(\bar{D})$ is in $H(\bar{D})$ if and only if there exists a neighborhood $\Omega$ of $E$ such that $\left.f\right|_{\bar{\Omega} \cap \bar{D}}$ is in $H(\bar{\Omega} \cap \bar{D})$.

Proof. Let $N_{1}$ be a small neighborhood of $\bar{D} \backslash \Omega$ and let $N_{2}$ be a neighborhood of $\bar{N}_{1}$ such that $N_{2} \cap \partial D \subset \subset s(\bar{D})$. Cover $\partial D \backslash \Omega$ by small balls $B_{1}, B_{2}, \ldots, B_{r} \subseteq N_{1}$ with centers $q_{1}, q_{2}, \ldots, q_{r} \in(\partial D \backslash \Omega)$. Let $\nu_{j}$ be the outward unit normal to $\partial D$ at $q_{j}$ and let $f_{j}^{\delta}(z)=f\left(z-\delta \nu_{j}\right)$. For sufficiently small $\underline{\delta}>0, f_{j}^{\delta}$ is holomorphic near $\bar{D} \cap \bar{B}_{j}$. Let $\tilde{D}$ be the neighborhood of $\bar{D}$ appearing in the lemma and set $B_{r+1}=D \backslash N_{1}$. Then $B_{1}, \ldots, B_{r}, B_{r+1}, \Omega$ is an open cover for $\bar{D}$. Let $\left\{\Phi_{1}, \ldots, \Phi_{r}, \Phi_{r+1}, \Phi_{0}\right\}$ be a partition of unity subordinate to this cover of $\bar{D}$. For $\varepsilon>0$ choose $\delta$ such that $\left\|f_{i}^{\delta}-f\right\|_{\bar{B}_{i} \cap \bar{D}}<\varepsilon$ for $i=1,2, \ldots, r$; choose a neighborhood $B_{0}$ of $\bar{\Omega} \cap \bar{D}$ and a function $f_{0} \in \operatorname{Hol}\left(B_{0}\right)$ with $\left\|f_{0}-f\right\|_{\bar{D} \cap \bar{\Omega}}<\varepsilon$. For $i=0,1, \ldots, r$ choose neighborhoods $\tilde{B}_{i}$ of $\bar{B}_{i} \cap \bar{D}$ such that $f_{i}$ is holomorphic on $\tilde{B}_{i}$ and $\left\|f_{i}-f_{j}\right\|_{\tilde{B}_{i} \cap \tilde{B}_{j}}<2 \varepsilon$ for $0 \leqslant i, j \leqslant r+1$ with $\tilde{B}_{r+1}=B_{r+1}$ and $f_{r+1}=f$. Let $D^{\prime}$ be a small $C^{(4)}$ perturbation of $D$ with $D^{\prime} \supset D \cup\left(\partial D \cap N_{2}\right)^{-}$and sufficiently small so as not to affect the constant $k$ appearing in the lemma.

Define $g_{i}=\sum_{\tilde{B} j=0}^{r+1} \Phi_{j}\left(f_{i}-f_{j}\right)$ on $\tilde{B}_{i}$ and observe that $\left\|g_{i}\right\|_{\tilde{B}_{i}}<2 \varepsilon, g_{i}-g_{j}=f_{i}$ $-f_{j}$ on $\tilde{B}_{i} \cap \tilde{B}_{j}$ and $g_{0} \mid \tilde{B}_{0} \backslash N_{1} \equiv 0$. Since $\bar{\partial} g_{i}=\bar{\partial} g_{j}$ on $\tilde{B}_{i} \cap \tilde{B}_{j}$, $\alpha=\bar{\partial} g_{j}$ 
defines a $C^{\infty}$ form of bi-degree $(0,1)$ on $\bigcup_{i=0}^{r+1} \tilde{B}_{i} \supseteq D^{\prime}$. By the lemma, there exists smooth $u$ on $D^{\prime} \cup\left(\tilde{D} \backslash N_{2}\right)$ such that $\partial u=\alpha$ on $D^{\prime}, \overline{\partial u}=0$ on $\tilde{D} \backslash N_{2}$ and $\|u\|_{\bar{D}} \leqslant k\|\alpha\|_{\bar{D}}$. But $\|\alpha\|_{\bar{D}} \leqslant C \varepsilon$ where $C$ depends only on the partition of unity $\left\{\Phi_{j}\right\}$. Letting $D_{\varepsilon}=D^{\prime} \cup\left(\tilde{D} \backslash N_{2}\right), h_{i}=g_{i}-u$ is holomorphic on $\tilde{B}_{i} \cap D$ and

$$
f_{i}-h_{i}=f_{i}-g_{i}+u=f_{j}-g_{j}+u=f_{j}-h_{j} \text { on } \tilde{B}_{i} \cap \tilde{B}_{j} \cap D_{\varepsilon},
$$

so $f_{\varepsilon}=f_{i}-h_{i}$ defines a holomorphic function on $D_{\varepsilon}$. Moreover,

$$
\begin{aligned}
\left\|f-f_{\varepsilon}\right\| & \leqslant \max _{0<i<r+1}\left\|f-f_{i}-h_{i}\right\|_{\tilde{B}_{i} \cap \bar{D}} \\
& =\max _{0<i<r+1}\left\|f-f_{i}+g_{i}-u\right\|_{\tilde{B}_{i} \cap \bar{D}} \\
& \leqslant \max _{0<i<r+1}\left\|f-f_{i}\right\|_{\tilde{B}_{i} \cap \bar{D}}+\left\|g_{i}\right\|_{\tilde{B}_{i} \cap \bar{D}}+\|u\|_{\bar{D}} \\
& \leqslant \varepsilon+2 \varepsilon+K C \varepsilon .
\end{aligned}
$$

The requirement that $f \mid \bar{\Omega} \cap \bar{D}$ be in $H(\bar{\Omega} \cap \bar{D})$ can be addressed by demonstrating the existence of a holomorphic vector field defined in a neighborhood of $E$ which is transverse to $\partial D$, as described by Fornaess and Nagel [16] and Bedford and Fornaess in [3] where some sufficient conditions for existence are derived. In particular, whenever $H(\partial D)=C(\partial D)$, an arbitrary smooth transverse vector field can be approximated by a holomorphic one.

There are various classes of weakly pseudoconvex domains which have been shown to be holomorphic sets; e.g., the regular domains of Diederich and Fornaess [10], [11], the domains of Weinstock [34], and the uniformly $H$-convex domains of Cirka [7]. Since both of the former types of domains are uniformly $\boldsymbol{H}$-convex, it may be appropriate to limit remarks to this latter class which includes bounded domains with real-analytic boundaries [13].

Definition 4.3. A compact set $K \subseteq \mathbf{C}^{n}$ is called uniformly $H$-convex if there exists a sequence of domains $G_{k} \supseteq K$ and a constant $C>0$ such that

$$
\frac{C}{k} \leqslant \operatorname{dist}\left(K, \partial G_{k}\right) \leqslant \sup _{z \in \partial G_{k}} \operatorname{dist}(K, z) \leqslant \frac{1}{k}
$$

for all $k$.

On domains $\Omega$ in $\mathbf{C}^{n}$ whose closures $\bar{\Omega}$ are uniformly $H$-convex, the algebra $H(\bar{\Omega})$ is dense in the algebra $A^{k+1}(\Omega) \equiv C^{(k)}(\bar{\Omega}) \cap \operatorname{Hol}(\Omega)$ with respect to the $C^{(k-n)}$ norm. Hence, these domains qualify as front-line assault points for enlarging the class of weakly pseudoconvex domains on which holomorphic approximation is possible.

5. Abstract holomorphic approximation. In the two types of counterexamples referred to in $\$ 1$, approximation failed either (a) because nonsmoothness of the boundary precluded local holomorphic approximation of restrictions of functions in the boundary value algebra near singularities, or (b) because homomorphism spaces do not coincide due to analytic continuation of functions in the smaller algebra.

With these two difficulties in mind, let $D$ be a relatively compact domain in $\mathrm{C}^{n}$, let $K$ be a compact subset of $\bar{D}$ containing $\partial_{0} H(\bar{D})$, and let $A_{K}(\bar{D})$ be the uniform algebra of uniform limits on $\bar{D}$ of functions which are continuous on 
$\bar{D}$ and which are holomorphic at each point of $\bar{D} \backslash K$. An abstract approximation theorem for the algebra $A_{K}(\bar{D})$ is obtained in [5]:

THEOREM 5.1. If $\bar{D}$ is holomorphically convex and if $\operatorname{Hol}(K)$ is dense in $A_{K}(\bar{D}) \mid K$, then $A_{K}(\bar{D})=H(\bar{D})$.

RemARK. In [5], Theorem 5.1 is proved for a holomorphic set $\bar{D}$ and the requirement that $K$ contain $\partial_{0} H(\bar{D})$ is inadvertently omitted in the statement of the theorem, but it is present in all of the applications cited therein. The motivating example was $\bar{D}$, a compact special analytic polyhedron, and $K$, the distinguished boundary of the polyhedron, on which every continuous function can be approximated by functions in $\operatorname{Hol}(K)$.

OUTLINE OF THE PROOF. Let $\mu$ be a measure orthogonal to $H(\bar{D})$ and supported on $K$ and let $g$ be a function in $A_{K}(\bar{D})$. Let $h \in H(\bar{D})$ approximate $g$ to within $\delta$ on a neighborhood $W$ of $K$ and let $U$ be an arbitrary neighborhood of $K$ contained in $W$. By smoothing, there exists a smooth function $g_{U}$ which approximates $g$ and is holomorphic where $g$ is off $U$. Choose a strictly pseudoconvex domain $\Omega$ contained in $W \cup\left\{z \in \mathbf{C}^{n}: \bar{\partial} g(z)\right.$ $=0$ \}. (For simplicity, assume here, as in [5], that $\bar{D}$ is a holomorphic set.) Let $\Phi$ be a smooth function such that $\Phi=1$ on $U, \Phi=0$ outside $W$ and $0<\Phi<1$. As described in $\$ 3$, an $(n, n-1)$ current $\lambda$ with measure coefficients supported in $\Omega$ can be found to satisfy $\bar{\partial} \lambda=\bar{\mu}$. Since $\bar{\partial} \Phi \lambda=\bar{\partial} \Phi$ $\wedge \lambda+\Phi \wedge \bar{\partial} \lambda$ and $\lambda$ has measure coefficients, $\partial \Phi \lambda$ is a measure as well, disjointly supported on $K$ and off $U$ in $W$. The part of this measure off $U$ annihilates all functions holomorphic on its support and the part of $\bar{\partial} \Phi \lambda$ supported on $K$ has total variation norm bounded by $\|\mu\|$, independent of the choice of $\delta$. Thus since

$$
\mu\left(g_{U}\right)=(\bar{\partial} \Phi \lambda+\bar{\partial}(1-\Phi) \lambda)\left(g_{U}\right)=\bar{\partial} \Phi \lambda\left(g_{U}\right)=\bar{\partial} \Phi \lambda\left(g_{U}-h\right)
$$

and

$$
\left|\bar{\partial} \Phi \lambda\left(g_{U}\right)\right|<\|\mu\|\left\|g_{U}-h\right\|_{W},
$$

it follows that $\mu\left(g_{U}\right)=0$ and, hence, that $\mu(g)=0$. Therefore, $H(\bar{D})=$ $A_{K}(\bar{D})$.

This result can be applied, in particular, when $\partial_{0} H(\bar{D})$ is contained in a stratified totally real set $M$, the disjoint union of sets $M_{j}, j=1,2, \ldots, k$, where each $M_{j}$ is a totally real (no complex tangents) $C^{(\infty)}$ submanifold of $C^{n} \backslash \cup_{i<j} M_{i}$. See Fornaess and Nagel [16].

When holomorphic convexity is not assumed, there should be criteria for deciding which functions in $A(\bar{D})$ are approximable by functions in $\operatorname{Hol}(\bar{D})$. Failure of approximation due to boundary singularities, presumably, is of diverse nature, but should be eliminated by hypothesizing local approximation. In the absence of boundary pathology, the lack of holomorphic convexity typically gives rise to analytic continuation. Recently, Sibony [29] has developed necessary and sufficient conditions for the existence of analytic structure of all possible dimensions in the homomorphism space of a uniform algebra and it would be tempting to conjecture the following: if every function in $A(\bar{D})$ can be locally approximated at each point of $\partial D$ by functions in $H(\bar{D})$, then $H(\bar{D}) \cong A(\Delta H(\bar{D}))$, where the latter algebra is understood to be all 
continuous functions on $\Delta H(\bar{D})$, also holomorphic on the analytic structure which is present in $\Delta H(\bar{D})$. The domain $\Omega_{1}$ of $\S 1$ is a case in point. However, is the conjecture verifiable even in the Diederich and Fornaess example $\Omega_{2}$ cited in the same section? One would like to say that precisely those functions in $A(\bar{D})$ which analytically continue to the same sets as functions in $H(\bar{D})$ can be approximated by functions in $H(\bar{D})$.

There are other approaches to this subject of equal importance which are neglected in the above discussion. Important among these are the work of Harvey and Lawson on holomorphic chains and their boundaries in [19], the work of Hörmander and Wermer [22] on uniform approximation on compact sets lying in smooth submanifolds in $\mathbf{C}^{n}$, and the work of Sibony and Wermer [31] on generators of the boundary value algebra.

Something could be said about holomorphic approximation in Stein manifolds and holomorphic approximation on arbitrary compact subsets of $\mathbf{C}^{n}$; by choice the discussion has been confined to approximation on the closure of relatively compact domains in $n$-dimensional complex space. For a more complete bibliography, the reader is referred to the survey paper [21] which lists 219 references to this general subject area.

ADDED IN PROOF. Without any assumption about the holomorphic convexity of $\bar{D}$, Beatrous [2] succeeded in showing that if $D$ is a weakly pseudoconvex domain in $\mathbf{C}^{n}$ with smooth boundary then any function in $A(\vec{D})$ can be uniformly approximated by functions in $A(\bar{D})$ which extend holomorphically across each strongly pseudoconvex boundary point of $D$. The essential ingredient of the proof is a version of Lemma 4.1 which yields solutions to inhomogeneous Cauchy-Riemann equations satisfying uniform and Hölder estimates and which is valid without assuming the holomorphic convexity of $\bar{D}$. The result then follows from a patching argument similar to the proof of Theorem 4.2.

\section{BIBLIOGRAPHY}

1. R. Arens, The maximal ideals of certain function algebras, Pacific J. Math. 8 (1958), 641-648. MR 22 \#8315.

2. F. Beatrous, Boundary value algebras, Dissertation, Tulane Univ., 1978.

3. E. Bedford and J. E. Fornaess, Domains with pseudoconvex neighborhood systems (to appear).

4. F. T. Birtel, Algebras of holomorphic functions, Tulane Lecture Note Series, 1972, pp. 1-100.

5. Mergelyan approximation on holomorphic sets, Bull. Inst. Math. Acad. Sinica 3 (1975), 183-190.

6. Some holomorphic function algebras, Papers from the Summer Gathering on Function Algebras, Matematisk Inst., Aarhus Univ., Aarhus, 1969, pp. 11-18.

7. E. M. Cirka, Approximation by holomorphic functions on smooth manifolds in $\mathbf{C}^{n}$, Mat. Sb. 78 (1969), 101-123 = Math. USSR-Sb. 7 (1969), 95-113.

8. B. Cole and R. M. Range, A-measures on complex manifolds and some applications, J. Functional Analysis 11 (1972), 393-400.

9. K. Diederich and J. E. Fornaess, Pseudoconvex domains: bounded strictly plurisubharmonic exhaustion functions, Invent. Math. 39 (1977), 129-141.

10. Pseudoconvex domains: An example with nontrivial Nebenhülle, Math. Ann. 225 (1947), 275-292.

11. , Pseudoconvex domains: Existence of stein neighborhoods, Duke Math. J. 44 (1977), 641-662. 
12. A strange bounded smooth domain of holomorphy, Bull. Amer. Math. Soc. 82 (1976),

$74-76$

13. Pseudoconvex domains with real-analytic boundary (to appear).

14. H. Federer, Geometric measure theory, Springer-Verlag, New York, 1969.

15. J. E. Fornaess, Embedding strictly pseudoconvex domains in convex domains, Amer. J. Math. 98 (1976), 529-569.

16. J. E. Fornaess and A. Nagel, The Mergelyan property for weakly pseudoconvex domains, Manuscripta Math. 22 (1977), 199-208.

17. T. W. Gamelin, Uniform algebras, Prentice-Hall, Englewood Cliffs, N.J., 1969.

18. M. Hakim and N. Sibony, Frontière de Shilov et spectre de $A(\bar{D})$ pour les domains failblement pseudoconvexes, C. R. Acad. Sci., Paris 281 (1975), 959-962.

19. F. R. Harvey and H. B. Lawson, Boundaries of complex varieties. I, Ann. of Math. (2) 102 (1975), 223-290.

20. G. M. Henkin, Integral representations of functions holomorphic in strictly pseudoconvex domains and some applications, Mat. Sb. 78 (120) (1969), 611-632 = Math. USSR Sb. 7 (1969), 597-616.

21. G. M. Henkin and E. M. Cirka, Boundary properties of holomorphic functions of several complex variables, J. Soviet Math. 5 (1976), 612-679.

22. L. Hörmander and J. Wermer, Uniform approximation on compact sets in $\mathbf{C}^{n}$, Math. Scand. 23 (1968), 5-21.

23. E. Kallin, Fat polynomially convex sets, Function Algebras, Scott, Foresman, Chicago, Ill., 1966, pp. 149-152. MR 33 \#2828.

24. N. Kerzman, Hölder and $L^{p}$ estimates for solutions of $\overline{\partial u}=f$ in strongly pseudoconvex domains, Comm. Pure Appl. Math. 24 (1971), 301-379. MR 43 \# 7658.

25. I. Lieb, Ein Approximationssatz auf streng pseudokonvexen Gebieten, Math. Ann. 184 (1969), $56-60$.

26. R. M. Range and Y. T. Siu, Uniform estimates for the $\bar{\partial}$-equation on domains with smooth strictly pseudoconvex boundaries, Math. Ann. 206 (1973), 325-354.

27. V. N. Senichkin, On the approximation of functions "of several complex variables on fat compact subsets of $C^{n}$ by polynomials, Mat. Sb. 97 (139) (1975), no. 2, 278-300 = Math. USSR Sb. 26 (1975), 260-279.

28. N. Sibony, Approximation de fonctions $\dot{a}$ valeurs dans un Fréchet, par des functions holomorphes, Ann. Inst. Fourier (Grenoble) 24 (1974), 167-179. MR 51 \#934.

29. Multidimensional analytic structure in the spectrum of a uniform algebra, Lecture Notes in Math., no. 512, Springer-Verlag, New York, 1976 (also appeared in Spaces of analytic functions, Kristiansand, Norway, 1975).

30. Un example de compact polynomialement convexe dans $\mathbf{C}^{2}$, Bull. Soc. Math. France 103 (1975), 141-147.

31. N. Sibony and J. Wermer, Generators for $A(\Omega)$, Trans. Amer. Math. Soc. 194 (1924), $103-114$.

32. A. G. Vitushkin, Analytic capacity of sets in problems of approximation theory, Russian Math. Surveys 22 (1967), 139-201. MR 37 \#5404.

33. B. Weinstock, Approximation by holomorphic functions on certain product sets in $\mathbf{C}^{n}$, Pacific J. Math. 43 (1972), 811-822.

34. __ Some conditions for uniform H-convexity, Illinois J. Math. 19 (1975), 400-404. MR 53 \# 13639.

35. R. M. Range, Holomorphic approximation near strictly pseudoconvex boundary points, Math. Ann. 201 (1973), 9-17.

Department of Mathematics, Tulane University, New Orleans, louisiana 70118 